\title{
Particle imaging velocimetry-based identification of coherent structures in normally impinging multiple jets
}

\author{
L. F. G. Geers ${ }^{\text {a) }}$ \\ Division 1-Physical Protection, TNO Prins Maurits Laboratory, P.O. Box 45, 2280 AA Rijswijk, \\ The Netherlands \\ M. J. Tummers and K. Hanjalić \\ Faculty of Applied Sciences-Thermofluids Section, Delft University of Technology, P.O. Box 5046, \\ 2600 GA Delft, The Netherlands
}

(Received 19 July 2004; accepted 8 March 2005; published online 29 April 2005)

\begin{abstract}
To gain a better insight into the effects of eddy structure and their thermal imprint on the impinging surface, we applied several methods to identify coherent structures in two arrangements of multiple jets. High-resolution particle imaging velocimetry measurements of the instantaneous fluid velocity have been performed to provide data for structure identification. Several methods based on critical point theory, i.e., vorticity magnitude, kinematic vorticity number, and second invariant of the velocity gradient tensor provided similar and generally useful information. However, in the flow considered they all appear inferior as compared with the proper orthogonal decomposition (POD). Despite lacking in conspicuous high energy modes, the flow showed to be well suited for POD, which provided clear identification of the near wall dominating vortical structure. (C) 2005 American Institute of Physics. [DOI: 10.1063/1.1900804]
\end{abstract}

\section{INTRODUCTION}

Impinging fluid jets are known to enhance strongly heat and mass transfers and are widely used for cooling, heating, and drying of solid surfaces. Industrial applications include quenching and tempering glass, annealing of metal and plastic, drying paper and textile, cooling of electronics, internal cooling of gas turbine blades, and others.

While the heat transfer intensification due to jet impingement is indisputable, its mechanism is still not fully clear, especially in multiple-jets configurations and if the flow is turbulent. Heat transfer under a single turbulent jet shows a large nonuniformity, with a maximum usually at the stagnation point. For short orifice-to-plate distances $(H / D \leqslant 2$, where $H$ is the orifice-to-plate distance and $D$ the jet diameter) and depending on the inflow turbulence level and jet Reynolds number, the maximum can be displaced somewhat further away from the stagnation point, or it can appear as a second peak in heat transfer coefficient. The heat transfer maximum is believed to be associated with surface renewal effect caused by large-scale eddies impinging on the surface, destroying the thermal boundary layer and rapidly convecting radially into the wall jets formed on the target surface after the jet deflection. ${ }^{1}$ This is further supported by strong turbulence from merging edge shear layers (if the distance from the orifice is sufficient for the potential core to disappear), as well as fast heat removal by the accelerating wall boundary layer. However, this simplistic picture does not envisage the precise role of turbulence and, especially, of coherent structures, associated with the edge vortices (toroidal

\footnotetext{
a) Telephone: +31 (0)15-2843405. Fax: +31 (0)15 - 2843963. Electronic mail: geers@pml.tno.nl
}

for round jets), as well as other large-scale structures within the jet.

The situation with multiple jets is further complicated by interaction between jets prior to their impingement. This interaction depends on jet configuration and their spacing, which appear as new factors that influence the heat transfer distribution. Secondary recirculation with an upwash fountain may appear in the space between the jets, together with some smaller embedded vortices, as well as cross flow of fluid around jets towards its exhaust. Multiple jets are usually aimed at making the heat transfer more uniform, but their interactions bring new elements of nonuniformity, reflecting the vortical structure in the wall vicinity.

The purpose of this work was to educe the vortical structures in an array of impinging jets, which could subsequently serve to explain the salient features of the jets' thermal signature on the target plate. Because of space limitations, we confine our presentation only to the structure identification in isothermal jets without considering heat transfer, the latter being the subject of another publication. The identification has been based on high-resolution PIV (particle imaging velocimetry). Two configurations have been considered and mutually compared: a hexagonal and an in-line jet arrangement. Several identification methods have been applied in order to find an optimum technique for this flow configuration, but most results to be presented have been obtained by proper orthogonal decomposition. We present first a brief outline of the identification techniques applied, followed by some information about the experiment. A comparative overview of educed coherent structures identified by various methods for the two jet configurations is then presented and discussed, followed by conclusions. 


\section{COHERENT STRUCTURES AND THEIR DETECTION}

Quasiperiodic, well organized, and repeating eddy formations, known as coherent structures are known to play a major role in turbulence dynamics and in transporting momentum, heat, and species in turbulent flows. Proper identification of such structures and their morphology is a major prerequisite for understanding their role in transport mechanisms. Of special interest are those regions in turbulent flows, such as impingement, separation, or reattachment regions, where the transport of scalars (heat and species) is markedly different from momentum transport, thus departing from the common Reynolds analogy, e.g., Bae and Sung. ${ }^{2}$ While in some cases a strong erratic movement of sweeping coherent structures can significantly enhance local heat transfer, stationary vortices can locally "trap" the fluid reducing the beneficial temperature or concentration difference and thus decreasing the heat or mass transfer. Therefore, adequate detection of these structures and their dynamics is of vital importance for the description of the flow and its effect on heat and mass transfer processes.

An intuitive way of detecting eddies is to search for spiraling or closed path lines or streamlines. However, the method is not Galilean invariant. This is one of two requirements for vortex identification mentioned by Jeong and Hussain. ${ }^{3}$ To exclude potential flow regions, the second requirement states that a vortex core must have a net vorticity (hence, net circulation). In this section we briefly outline a number of methods for structure identification and their characteristics, which we applied for analyzing the coherent structure in multiple impinging jets.

Vorticity magnitude. The magnitude of the vorticity vector $\|\boldsymbol{\omega}\|$ is widely used to identify coherent structures. Equation (1) shows its definition:

$$
\|\boldsymbol{\omega}\|=\left\{\sum_{i=1}^{3}\left(\epsilon_{i j k} \frac{\partial u_{k}}{\partial x_{j}}\right)^{2}\right\}^{1 / 2},
$$

where $\epsilon_{i j k}$ is the alternating unit tensor, $x_{j}$ is the $j$ th coordinate, and $u_{k}$ is the velocity component in the $x_{k}$ direction. Although the use of $\|\boldsymbol{\omega}\|$ is successful in free shear flows, it does not always produce satisfactory results in flows near obstacles or along walls. ${ }^{3}$ The vorticity magnitude is not only sensitive to the local swirling motion typical for a vortex, but also to shear. So, when using the vorticity magnitude as an indication for turbulent structures, it is not easy to distinguish true vortices from background shear, when the shear is relatively high. A high value of the vorticity magnitude is a necessary but insufficient condition for detecting a vortex.

Second invariant of the velocity gradient tensor. The identification method on the basis of $Q$, the second invariant of the velocity gradient tensor, was originally proposed by Hunt et al. ${ }^{4} Q$ can be interpreted as a measure for the magnitude of rotation relative to strain and is defined as follows:

$$
Q \equiv-\frac{1}{2} \frac{\partial u_{i}}{\partial x_{j}} \frac{\partial u_{j}}{\partial x_{i}}=\frac{1}{2}\left(\|\mathbf{\Omega}\|^{2}-\|\boldsymbol{S}\|^{2}\right),
$$

where $\boldsymbol{S}$ and $\boldsymbol{\Omega}$ are the symmetric and antisymmetric components of the velocity gradient tensor. When $Q$ is positive, rotation prevails over strain so that vortices can be identified as positive values of $Q$. The advantage of $Q$ over the vorticity magnitude as a quantitative vortex identifier is the fact that $Q$ represents the local balance between shear strain rate and vorticity magnitude. Hence, $Q$ is not affected by local shear, which usually prevails in the vicinity of walls.

Kinematic vorticity number. An identifier similar to $Q$ is the kinematic vorticity number $N_{k}$. This quantity is equal to the magnitude of the vorticity vector nondimensionalized by the strain rate and can be related to $Q$ :

$$
N_{k} \equiv \sqrt{\frac{\|\boldsymbol{\omega}\|^{2}}{2 S_{i j} S_{i j}}}=\sqrt{1+\frac{2 Q}{S_{i j} S_{i j}}},
$$

where

$$
S_{i j} \equiv \frac{1}{2}\left(\frac{\partial u_{i}}{\partial x_{j}}+\frac{\partial u_{j}}{\partial x_{i}}\right) .
$$

For example, $N_{k} \rightarrow \infty$ and $N_{k}=0$ correspond to solid-body rotation and irrotational motion, respectively, regardless of the vorticity magnitude. A region with $N_{k}>1$ is identical to that with $Q>0$. A disadvantage is that $N_{k}$ does not discriminate between vortices with small and large vorticity as long as the quality of rotation is the same for both. ${ }^{3}$

Other methods based on the velocity gradient tensor. Identification of vortices on the basis of a negative second eigenvalue $\left(\lambda_{2}\right)$ of $S^{2}+\boldsymbol{\Omega}^{2}$ forms a fourth identification method based on the velocity gradient tensor. Identification on the basis of the $Q$ definition may be incorrect when vortices are subjected to a strong external strain, whereas the $\lambda_{2}$ definition represents vortices correctly in this case. This method is superior to the $Q$ method described above as argued in details by Jeong and Hussain. ${ }^{3}$

Chong et al. ${ }^{5}$ describe the use of the eigenvalues of the velocity gradient tensor for structure identification. This method is known as the critical-point analysis. A criticalpoint is a point in the flow field where all three velocity components are zero and the streamline slope is indeterminate. These points can be found by calculating the eigenvalues of the velocity gradient tensor. An eddy is characterized by one real eigenvalue and a pair of complex conjugate eigenvalues with a positive imaginary part. Critical-point analysis also correctly identifies vortices subjected to strain. It should be noted that the $Q$ method, the $\lambda_{2}$ method, and the critical-point analysis are equivalent methods in planar flows (i.e., two-dimensional flows). Hence, the advantages of the $\lambda_{2}$-method and critical-point analysis over the $Q$ method only emerge in three-dimensional flows.

\section{HIGH FREQUENCY NOISE REMOVAL}

The above-mentioned structure identification mechanisms are all based on spatial derivatives of the fluid velocity. The calculation of these derivatives implies a certain continuity and smoothness of the velocity field. However, velocity fields resulting from PIV measurements are discrete in space and contain noise, so these demands are hard to meet. Additionally, high frequency noise in a signal is amplified by taking the derivative of this signal. For these rea- 
sons it is better to first filter the signal with a low-pass filter before derivatives are calculated for structure identification purposes.

Homogeneous filtering techniques, for instance, using a Gaussian kernel, provide a fast way of damping or removing high frequency noise. However, one of the problems with using homogeneous filtering in a turbulent flow field that has statistically inhomogeneous directions is that the character of the filter does not change as a function of the inhomogeneous coordinate. ${ }^{6}$ In case of strong shear layers, for instance, the width of the low-pass filter should grow as a function of the distance from the shear layer to reflect the fact that the turbulent eddies increase in size as one moves away from the shear layer. This problem can be addressed by using the method of proper orthogonal decomposition (POD) to construct inhomogeneous low-pass filters. When applied to experimental data, the POD can be viewed as a filtering device used to objectively eliminate the low energy motions of the flow that are obscuring the main energetic features of the flow. ${ }^{7}$ A brief description of the POD used in this research is given below. A more complete analysis can be found, e.g., in Manhart and Wengle. ${ }^{8}$

A velocity field $u(x, t)$ can be expanded into an infinite series of orthogonal spatial basis functions $\boldsymbol{\varphi}^{n}(\boldsymbol{x})$ and uncorrelated coefficients $a^{n}(t)$, where $n$ denotes the mode number:

$$
u(\boldsymbol{x}, t)=\sum_{n=0}^{\infty} a^{n}(t) \boldsymbol{\varphi}^{n}(\boldsymbol{x}) .
$$

Mathematically, the POD projects the random velocity $u(\boldsymbol{x}, t)$ into an orthonormal coordinate system $\boldsymbol{\varphi}^{n}(\boldsymbol{x})$. The projection is optimal in the sense that the first projection $(n=0)$ captures most of the energy; the energy contribution of subsequent modes decreases with increasing mode number. By considering only the modes comprising the most energetic components of the velocity signal, the series representation in Eq. (5) can be restricted to only $N_{M}$ modes. In other words, $N_{M}$ is the number of modes required to represent the flow field in a sufficiently accurate way, which is to be determined for each flow problem separately.

When POD is applied to an ensemble of experimental velocity fields consisting of instantaneous snapshots of the flow field that are uncorrelated in time, the temporal coefficients can be found by using the method of snapshots proposed by Sirovich. ${ }^{9}$ The energy contribution of each separate mode to the total energy is given by the eigenvalue $\lambda$ of the two-point temporal correlation tensor $C\left(t, t^{\prime}\right)$ for that particular mode:

$$
\int_{T} C\left(t, t^{\prime}\right) a^{n}\left(t^{\prime}\right) d t^{\prime}=\lambda^{n} a^{n}(t),
$$

in which $T$ is the time period in which the snapshots of the flow field are acquired, and $C\left(t, t^{\prime}\right)$ is defined as

$$
C\left(t, t^{\prime}\right)=\frac{1}{T} \iiint_{V} u(\boldsymbol{x}, t) \cdot u\left(\boldsymbol{x}, t^{\prime}\right) d \boldsymbol{x} .
$$

$V$ is the domain where the flow velocities are measured.

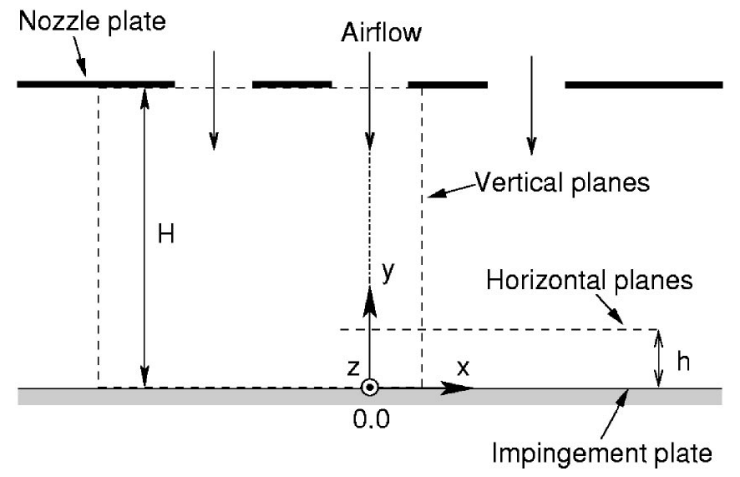

FIG. 1. Flow configuration for both the hexagonal and the in-line jet array.

When the snapshots of the flow are independent from each other, the time averaging integral in Eq. (6) can be replaced by an ensemble averaging sum.

\section{EXPERIMENTS}

Well defined boundary conditions are crucial to the investigation of the flow of an impinging jet array. All jets in the array should exhibit the same initial velocity profile and turbulence levels. Additionally, the jets must be swirl-free, implying a swirl-free flow upstream of the orifice plate. A wind tunnel was constructed that met these demands. It was designed on the basis of rules of thumb devised by Mehta and Bradshaw. ${ }^{10}$

Figure 1 shows the flow configuration for the jet array flow measurements. Air from the wind tunnel was issued through an orifice plate, after which it impinged on an impingement plate parallel to the orifice plate. For this purpose a glass plate was used to enable PIV velocity measurements in planes parallel to the impingement plate. Two different orifice plates were tested: one with 13 orifices with a diameter $D_{m}$ of $13 \mathrm{~mm}$ in a hexagonal arrangement and one with 9 orifices with the same diameter in an in-line arrangement (Fig. 2). The orifice pitch was $2 D_{m}$ for the hexagonal arrangement and $4 D_{m}$ for the in-line arrangement. Measurements were conducted using a distance between the orifice plate and the impingement plate $H$ of $4 D_{m}$ for both orifice configurations.

In the hexagonal orifice configuration measurements were conducted in three planes parallel to the jets (the vertical planes 1-3), as shown in Fig. 2(a). Vertical plane 1 intersects the central jet and one of its direct neighbors, vertical plane 3 intersects the central jet and one of the outer jets, and vertical plane 2 is placed along the bisector of planes 1 and 3 . The origin of a $x, y, z$-coordinate system is at the intersection of the centerline of the central jet and the surface of the impingement plate. The $y$ axis is measured along this centerline and assumed to be positive in the upward direction. The $x$ and $z$ axes are measured along the surface of the impingement plate. The measurement area was defined by -4.4 $<x / D_{m}<1.0$ and $0.0<y / D_{m}<4.0$ for all vertical planes in the hexagonal configuration.

Next to that, velocity measurements were performed in the same configuration in one plane parallel to the impingement plate (the horizontal plane), at a distance $h$ of $0.25 D_{m}$ 


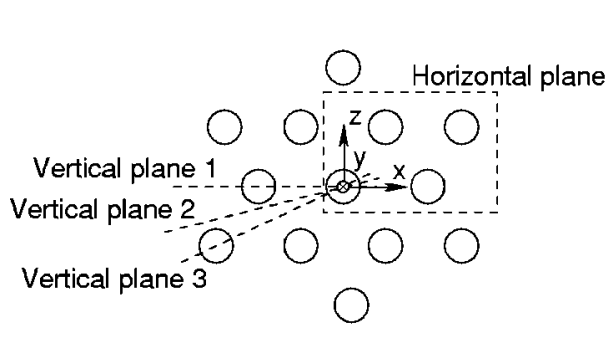

(a) hexagonal configuration

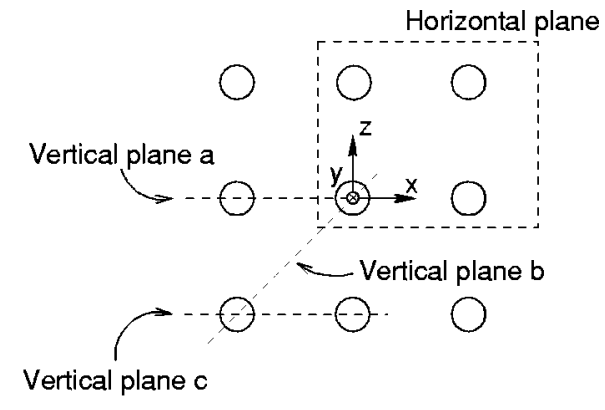

(b) in-line configuration
FIG. 2. The orientation of the measurement planes with respect to the orifice plates. above the impingement plate. This plane focuses on the same four jets as the vertical planes: the central jet, two of its direct neighbors and one of the outer jets. The position of the measurement area was $-0.6<x / D_{m}<3.5$ and $-0.7<z / D_{m}$ $<2.6$ in the hexagonal configuration.

In the in-line orifice configuration measurements were done in three vertical planes (planes $a-c$ ) depicted in Fig. 2(b). Plane $a$ intersects the central jet and one of its direct neighbors, plane $b$ intersects the central jet and one of the outer jets, and plane $c$ intersects one of the central jet's direct neighbors and one of the outer jets. The measurement area was defined by $-1.2<x / D_{m}<5.0$ and $0.0<y / D_{m}<4.0$ for vertical planes $a$ and $c$, and $-0.7<x / D_{m}<6.6$ and 0.0 $<y / D_{m}<4.0$ for vertical plane $b$.

Finally, measurements were done in one horizontal plane in the in-line configuration at $h=0.23 D_{m}$. This plane focuses on the same four jets as the vertical planes: the central jet, two of its direct neighbors, and one of the outer jets. The position of the measurement area was $-1.9<x / D_{m}<5.3$ and $-0.8<z / D_{m}<5.0$.

The impingement flow was investigated using PIV. The PIV system [manufactured by Optical Flow Systems (OFS)] included a double pulsed Nd:YAG (YAG-yttrium aluminum garnet) laser (Continuum Minilite) with a pulse energy of $25 \mathrm{~mJ}$. This laser was used to produce a $1 \mathrm{~mm}$ thick sheet that illuminated the flow. A PCO Sensicam camera with a resolution of $1280 \times 1024$ pixels recorded images of the seeding particles in the laser sheet. The commercial software VIDPIV ROWAN V4.0 developed by OFS/ILA was used to analyze the images.

The seeding consisted of an aqueous glycerol solution and was produced by a Laskin orifice. For the measurements in the vertical planes the laser produced a light sheet perpendicular to the impingement plate and the orifice plate and the camera recorded images of particles in the light sheet from the side. For the horizontal planes the light sheet was parallel to both the impingement plate and the orifice plate, and the camera recorded images from the bottom, through the glass impingement plate.

Table I presents the recording parameters of the PIV system for the four different configurations. As can be seen in Table I, the interrogation areas in the horizontal planes in both arrays were twice the size of the interrogation areas in the vertical planes. Because the noise level in the PIV images in the horizontal planes was higher than that in the vertical planes, more particle images were needed for a good signalto-noise ratio. The reason for the higher noise level is the fact that the images were taken through the impingement plate

TABLE I. Characteristics of the PIV measurements for all configurations.

\begin{tabular}{|c|c|c|c|c|}
\hline & \multicolumn{2}{|c|}{ Hexagonal jet array } & \multicolumn{2}{|c|}{ In-line jet array } \\
\hline & Horizontal & Vertical & Horizontal & Vertical \\
\hline Focal length lens (mm) & 55 & 105 & 55 & 55 \\
\hline Numerical aperture & 8 & 11 & 8 & 8 \\
\hline Field of view $\left(\mathrm{mm}^{2}\right)$ & $53.3 \times 42.9$ & $71.2 \times 52.0$ & $85.2 \times 66.2$ & $75.5 \times 52.0$ \\
\hline Interrogation area size (pixels) & $64 \times 64$ & $32 \times 32$ & $64 \times 64$ & $32 \times 32$ \\
\hline$\left(\mathrm{mm}^{2}\right)$ & $2.7 \times 2.7$ & $1.8 \times 1.8$ & $4.7 \times 4.7$ & $\begin{array}{l}2.0 \times 2.0^{\mathrm{a}} \\
2.4 \times 2.4^{\mathrm{b}}\end{array}$ \\
\hline Interrogation area overlap & $50 \%$ & $50 \%$ & $50 \%$ & $50 \%$ \\
\hline Pulse delay $(\mu \mathrm{s})$ & 11.7 & 20.0 & 10.0 & $\begin{array}{l}21.8^{\mathrm{a}} \\
25.8^{\mathrm{b}}\end{array}$ \\
\hline Dynamic velocity range $(\mathrm{m} / \mathrm{s})$ & $0.36: 57$ & $0.28: 22$ & $0.73: 116$ & $0.29: 23$ \\
\hline Relative error & $1.7 \%$ & $1.4 \%$ & $3.2 \%$ & $1.3 \%$ \\
\hline Reynolds number & $18 \times 10^{3}$ & $18 \times 10^{3}$ & $20 \times 10^{3}$ & $20 \times 10^{3}$ \\
\hline Number of snapshots & 1000 & 3000 & 3000 & 3000 \\
\hline
\end{tabular}

${ }^{\mathrm{a}}$ Planes $a$ and $c$.

${ }^{\mathrm{b}}$ Plane $b$. 


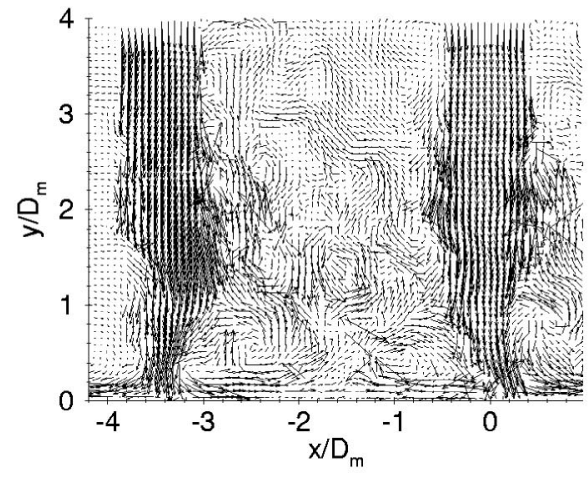

(a) Snapshot of velocity field

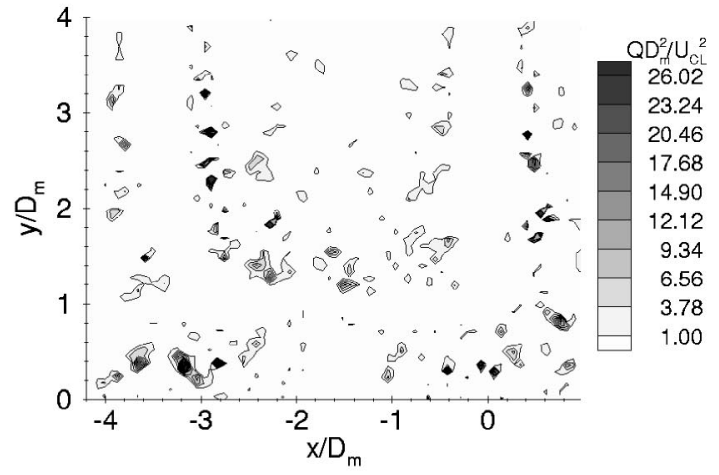

(c) $2^{\text {nd }}$ invariant of velocity gradient $Q$

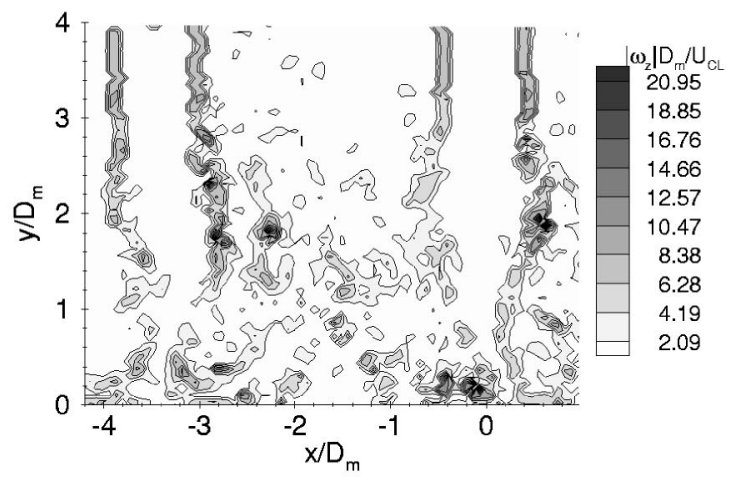

(b) Vorticity magnitude $\left|\omega_{z}\right|$

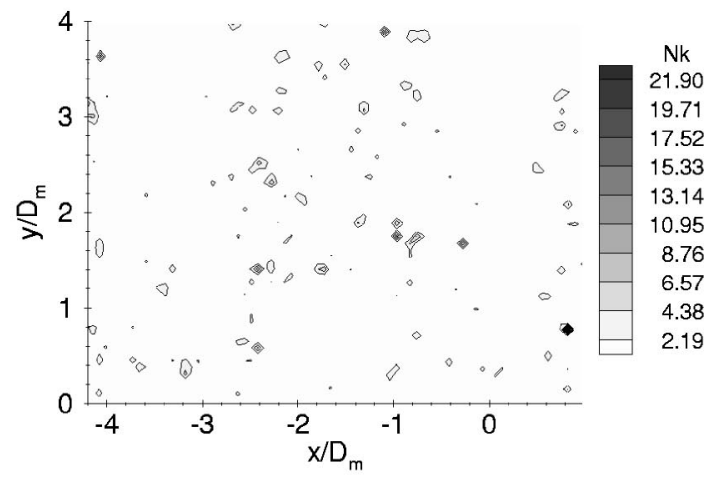

(d) Kinematic vorticity number $N_{k}$

FIG. 3. A snapshot of the velocity field in vertical plane 3 for the hexagonal configuration and plots of $\left|\omega_{z}\right|, Q$, and $N_{k}$.

which was gradually fouled by seeding deposition. Between every 200 images the impingement plate had to be cleaned. The dynamic range and the relative error (based on the jet's centerline velocity) of the velocity measurements are also given in the table. For the quantities derived from the velocity field it was calculated that the relative error of both the vorticity magnitude and $Q$ is twice as large as the error of the velocity, and the relative error of $N_{k}$ is $\sqrt{6}$ times as large.

Additionally, it can be seen that the number of snapshots in the ensemble is only 1000 for the horizontal planes of the hexagonal jet array, whereas it is 3000 for the other configurations. Cleaning of the impingement plate took a large amount of time. After these measurements the cleaning system was automated and sped up, so more measurements could be done in the same time.

The images resulting from all configurations were analyzed in three consecutive steps. First, the interrogation areas were cross correlated and a local median filter was used to discard the spurious vectors. The resulting empty spaces were filled with interpolated values from the surrounding interrogation areas. The resulting displacement fields were used as window displacements for an adaptive cross correlation of the same interrogation areas in the second step. After filtering out and replacing the spurious vectors the second step is repeated. The percentage of spurious vectors was $4 \%$ on average for all vertical planes and about $3 \%$ for the horizontal planes. The motivation for repeating the second step is a significant reduction of the number of spurious vectors.

\section{RESULTS}

On the basis of POD filtered snapshots of the flow in both configurations described above, details on the flow characteristics of impinging jet arrays is extracted. The structure identification methods described in Sec. II were applied to the experimental velocity data obtained with PIV. The quality of the methods will be assessed on the basis of the unfiltered velocity fields. Next, the method that proved most adequate is used to extract the largest most-energetic structures for PIV velocity data by first applying a POD filter before structures are identified. Once vortices are identified, they are located and sized in order to investigate the positions of eddies of different sizes.

\section{A. Structure identification methods}

Because the PIV system used in the present investigation only produced two-dimensional velocity fields for two components of the velocity vector, two-dimensional representations of the vorticity vector magnitude $\|\boldsymbol{\omega}\|$, the second invariant of the velocity gradient tensor $Q$, and the kinematic vorticity number $N_{k}$ are used. Instead of the magnitude of the vorticity vector $\boldsymbol{\omega}$, the absolute value of the vorticity vector's out-of-plane component is used. $Q$ and $N_{k}$ are calculated from the two-dimensional velocity gradient tensor. A secondorder central differencing scheme was chosen for the approximation of the gradients to maintain high resolution, while having an acceptable noise sensitivity. 


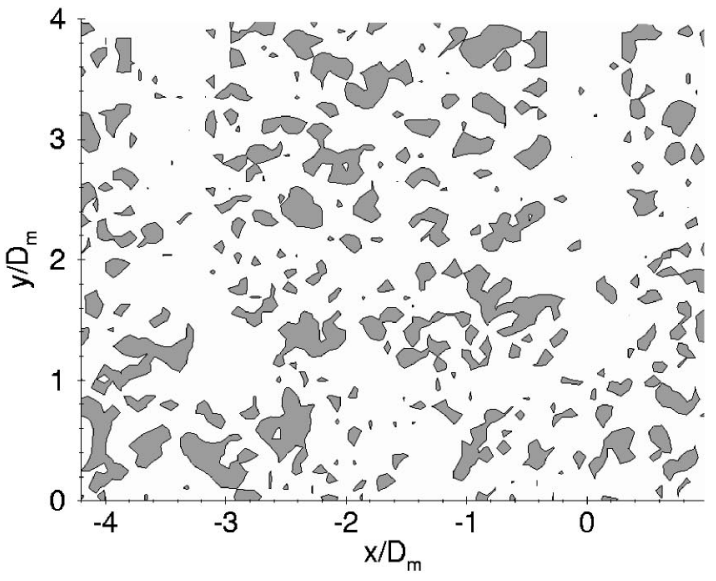

(a) $Q D_{m}^{2} / U_{C L}^{2}=0.0$

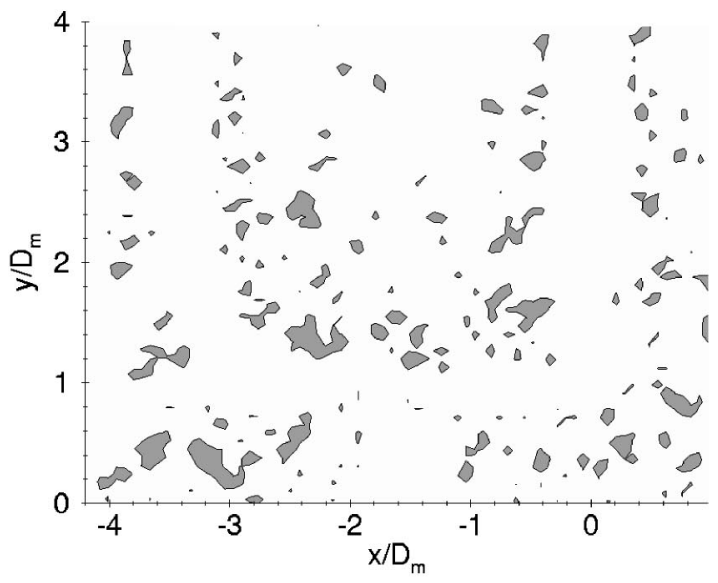

(c) $Q D_{m}^{2} / U_{C L}^{2}=1.0$

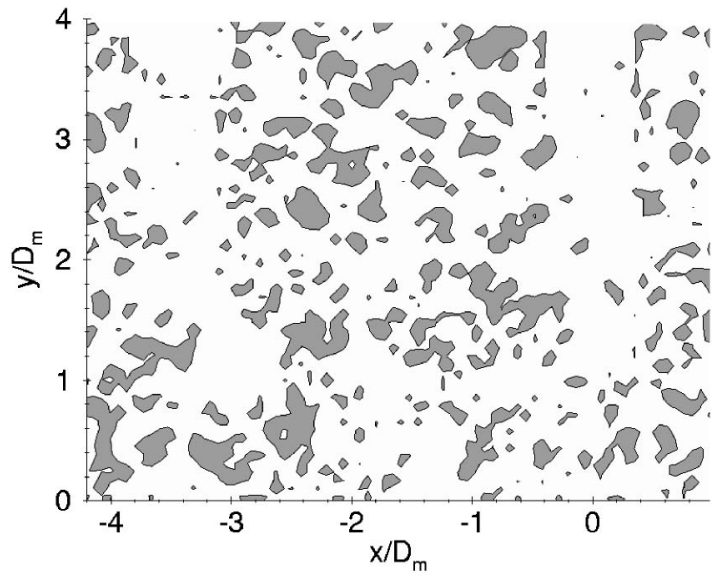

(b) $N_{k}=1.0$

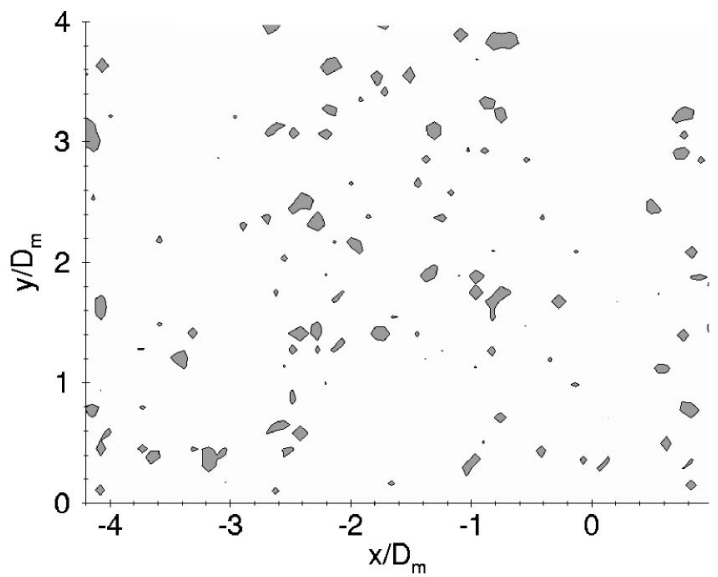

(d) $N_{k}=2.0$

FIG. 4. Comparison of vortical structures in plane 3 of the hexagonal configuration for different values of $Q$ and $N_{k}$.

Figure 3(a) presents the velocity vectors for a single realization of the flow field in plane 3 of the hexagonal configuration. The center jet and one of the outer jets can be identified. Structures of different sizes and shapes strongly affect the jets, mainly in the impingement region. Vortex cores with a (nearly) zero convection velocity with respect to the fixed reference frame of the snapshot can be identified. Moving or distorted vortices are harder to identify by eye.

Figure 3(b) shows the $\left|\omega_{z}\right|$ map associated with the velocity snapshot. The vorticity magnitude is nondimensionalized by the orifice diameter and the centerline velocity of the jets. The vorticity magnitude is large in the strong mixing layer directly downstream of the orifice edges. However, it is not possible to identify the more or less regular arrays of toroidal vortices that are formed in this region, as can be seen in Fig. 3(a). The reason for this is that $\left|\omega_{z}\right|$ is not only sensitive to the local swirling motion typical for a vortex, but also to shear. Further downstream of the orifices isolated regions with a high vorticity are found, some of them coincide with the locations of the vortices visible in the velocity plot.

Figure 3(c) shows the values of $Q$ computed from the instantaneous velocity field of Fig. 3(a). $Q$ is nondimension- alized with the orifice diameter and the centerline velocity. In comparison to the vorticity magnitude, a $Q$ map better identifies the individual toroidal vortices that form in the mixing layers downstream of the orifices. Distinct islands of high $Q$ are found, even in the shear layers directly downstream of the orifices.

A plot of the kinematic vorticity number is presented in Fig. 3(d). Since $N_{k}$ is nondimensionalized by the magnitude of the strain rate, the peak $N_{k}$ value is oblivious of the dynamical significance of a vortex. ${ }^{3}$ In other words, $N_{k}$ does not discriminate between vortices with small vorticity in a flow with small shear and vortices with large vorticity in a flow with large shear. This can be seen clearly when the $N_{k}$ map is compared to the $Q$ map. Vortices identified with similar $N_{k}$ values can have very different $Q$ values. This is even more clear from Fig. 4. In this figure regions are plotted where $Q$ and $N_{k}$ exceed a certain threshold level. On the left the $Q$ maps are presented and on the right the $N_{k}$ maps. For the lowest threshold values [Figs. 4(a) and 4(b)] both methods yield exactly the same results. This follows directly from the relationship between $N_{k}$ and $Q$ presented in Eq. (3).

In Figs. 4(c) and 4(d) the results are presented using higher threshold values. In Fig. 4(c) structures are identified 


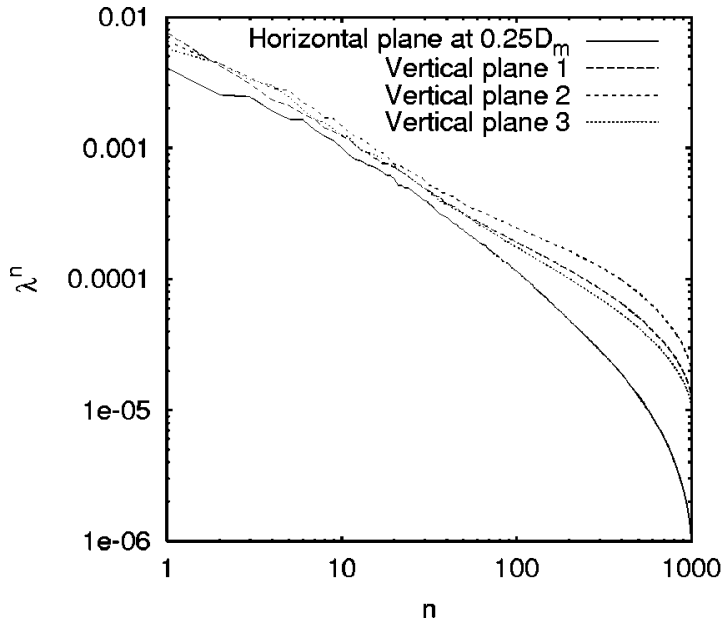

(a) Hexagonal configuration

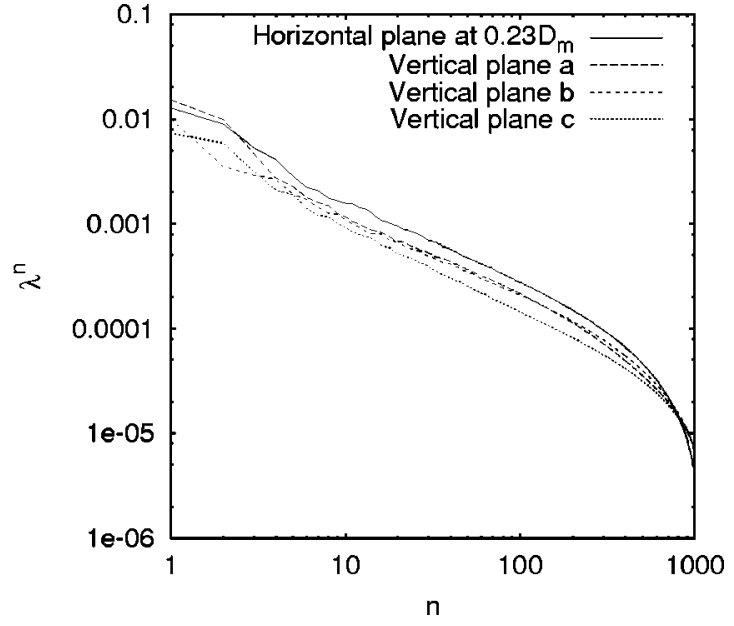

(b) In-line configuration

FIG. 5. POD eigenvalue spectra for vertical and horizontal planes in both the hexagonal and the in-line configuration.

with different sizes and shapes. The toroidal vortices in the shear layers of the jets can be discerned clearly and the largest structures appear near the impingement plate and in the region between the jets, where the upwash is located. In between the jets, towards the orifice plate only some smaller structures appear, because this region is relatively quiescent as can also be seen in the snapshot of the velocity field [Fig. 3(a)]. However, using $N_{k}$ structures are identified outside the shear layers and everywhere in between the jets, but none is identified inside the shear layers. In the snapshot of Fig. 3(a) the fluid appears to be almost stagnant in this zone and no clear vortices are visible there.

\section{B. Proper orthogonal decomposition}

The eigenvalue spectrum of a POD can be constructed by plotting eigenvalues versus modes. To check the effect of sample size on eigenvalue spectra, PODs were calculated for ensembles of different sizes taken from a single data set. It appeared that there was no significant difference between the spectra for the first 50 modes, when the sample size was over 1000. For the hexagonal configuration PODs were calculated for all three vertical planes and for the horizontal plane. For the in-line configuration PODs were also calculated for all three vertical planes and for the horizontal plane. For all planes the ensemble size was 1000.

The spectra depicted in Fig. 5 show the eigenvalues of the PODs for the vertical and the horizontal planes of the hexagonal and in-line configurations. The eigenvalues of all modes $\lambda^{n}$ are plotted against the mode number $n$ on a double logarithmic scale. All spectra show a very gradual decrease of energy going to higher POD modes and they extend over a large range of modes. The high Reynolds number will cause a very wide spectrum of turbulent kinetic energy, which means there is energy in structures of a wide range of scales. Therefore, a considerable number of modes should be taken into account to cover the larger part of the turbulent kinetic energy.

Table II gives an idea of how the energy is distributed over the first few modes for all measurements. The percentages are summed values for the energy contribution to the ensemble of the structures in groups of ten modes. The energy of mode 0 , representing the average velocity field, is not taken into account; the presented percentages refer to the fluctuations only. It is clear that roughly one-third of the total

TABLE II. Percentages of spatial energy per ten modes for vertical and horizontal planes in the hexagonal and the in-line configurations.

\begin{tabular}{llccccc}
\hline \hline \multirow{4}{*}{ Hexagonal } & Modes & $1-10$ & $10-20$ & $20-30$ & $30-40$ & $40-50$ \\
& Vertical 1 & 23.6 & 7.7 & 5.1 & 3.7 & 3.0 \\
& Vertical 2 & 19.8 & 6.9 & 4.4 & 3.3 & 2.8 \\
& Vertical 3 & 25.4 & 8.6 & 5.5 & 4.0 & 3.2 \\
\multirow{4}{*}{ In line } & Horizontal & 30.5 & 11.4 & 7.4 & 1.8 & 0.1 \\
& Vertical $a$ & 34.4 & 6.9 & 4.8 & 3.8 & 3.2 \\
& Vertical $b$ & 25.6 & 7.3 & 5.1 & 4.0 & 3.4 \\
& Vertical $c$ & 29.9 & 7.7 & 5.1 & 3.8 & 3.2 \\
& Horizontal & 29.2 & 8.0 & 5.5 & 4.2 & 3.5 \\
\hline \hline
\end{tabular}




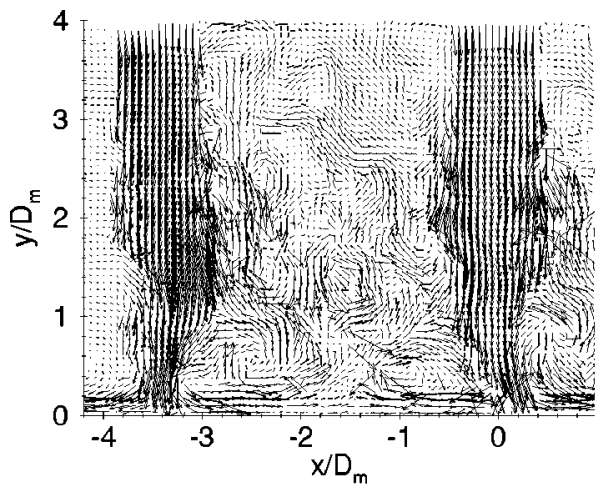

(a) Original snapshot

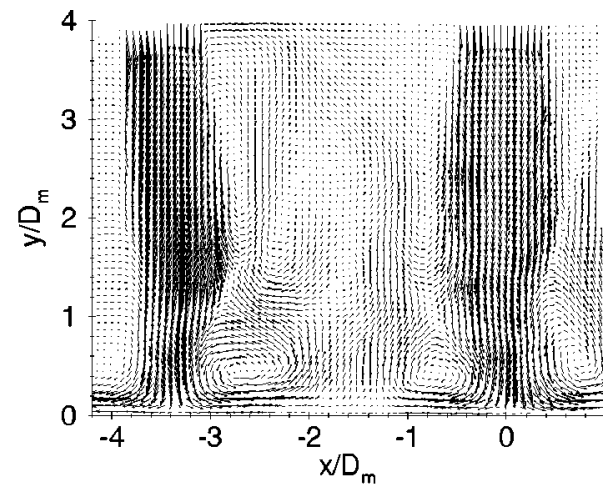

(b) POD filtered snapshot
FIG. 6. Example of a snapshot in plane 3 of the hexagonal configuration and its POD filtered equivalent (20 modes + mode 0 ). energy of the fluctuations is contained in the first twenty modes of the POD for all cases. Figure 6 presents the same snapshot as the one in Fig. 3 and its POD filtered equivalent using the first 21 modes including mode zero to see the effect of the large scale disturbances on the ensemble averaged flow field. The POD filtered snapshot shows the large scale eddies present around the jets and their impingement positions, while these structures appear masked or deformed in the original snapshot. Because the energy content of the modes above mode 20 is very low, the large scale features of the velocity fields are well represented by the first 20 modes.

\section{PODs of the hexagonal configuration}

Figure 7 presents two randomly picked snapshots of the flow in vertical plane 1 of the hexagonal configuration. These snapshots are reconstructed from the POD using the mean field (mode 0 ) and the first 20 modes to investigate only the large scale features of the flow. The center jet and its direct neighbor can be discerned clearly. They appear to be largely unaffected down to $y=2 D_{m}$, but below this position the jets are disturbed by eddies originating from the interaction of the jets with the impingement surface. For instance, in Fig. 7(b) the center jet is either broken up or severely displaced out of plane.

The upwash flow between the two jets is strongly affected by eddies near the impingement point of the two jets. In Fig. 7(a), for instance, the upwash is directed towards the neighbor jet due to an eddy near the neighbor jet.
Figure 8 shows two POD filtered snapshots at vertical plane 2 of the hexagonal configuration. The center jet is clearly visible again. Because this plane is positioned very near a neighbor jet, its influence on the surrounding air can be recognized by the relatively strong downflow around $x=-2 D_{m}$. Additionally, the upwash caused by colliding wall jets is visible. Two discharge mechanisms can be recognized in Figs. 8(a) and 8(b); part of the upwash flows outwards along the orifice plate avoiding the adjacent jets, while another part is being engulfed in the downflow of the neighbor jet. $^{11}$

Figures 8(a) and 8(b) show the horse-shoe vortex induced by the wall jet of the nearest outer jet and the spent air flowing outwards. This vortex also appears in Fig. 9. This figure presents two snapshots in plane 3 of the hexagonal configuration. The vortex does not have a steady position near the outer jet, which explains the fact that the ensemble mean velocity field also shows this vortex, but less clearly.

In Fig. 9(b) a large elongated eddy is formed in the shear layer of the outer jet at $\left(x / D_{m}, y / D_{m}\right) \approx(2.8,1.4)$. As was mentioned above, the upwash amplifies the instabilities in the shear layer of the jets, resulting in this kind of eddies. On the other side of the outer jet no upwash is present, so the shear layer on that side is not producing such eddies. For this reason the kinetic energy of turbulence $k$ is asymmetrically distributed around the outer jets, as was reported by Geers et $a l .{ }^{12}$ All figures of the POD filtered snapshots in the vertical

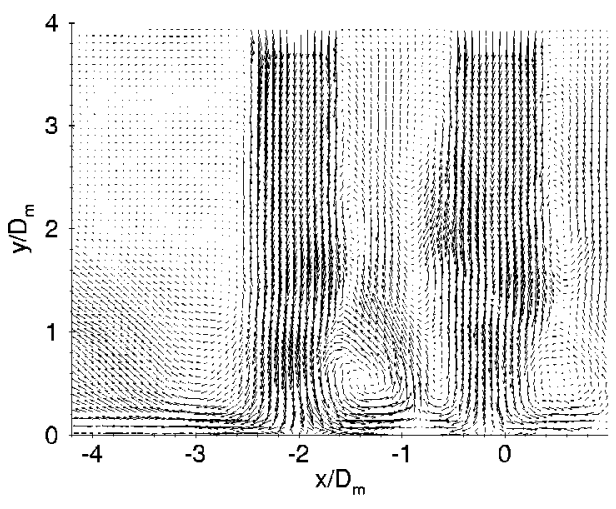

(a) snapshot 1

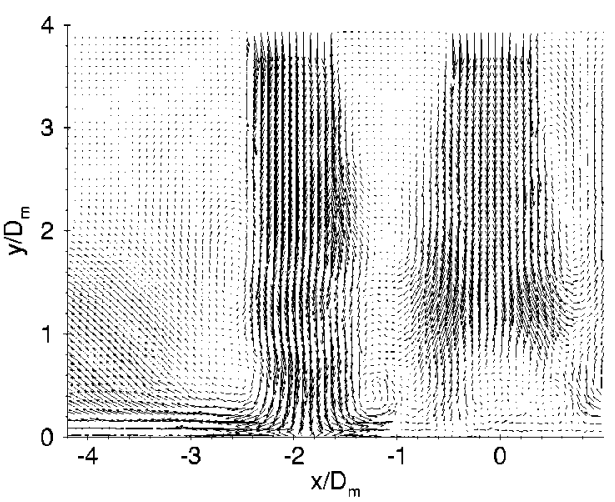

(b) snapshot 2
FIG. 7. POD filtered snapshots (mean field and the first 20 modes) in vertical plane 1 of the hexagonal arrangement. 


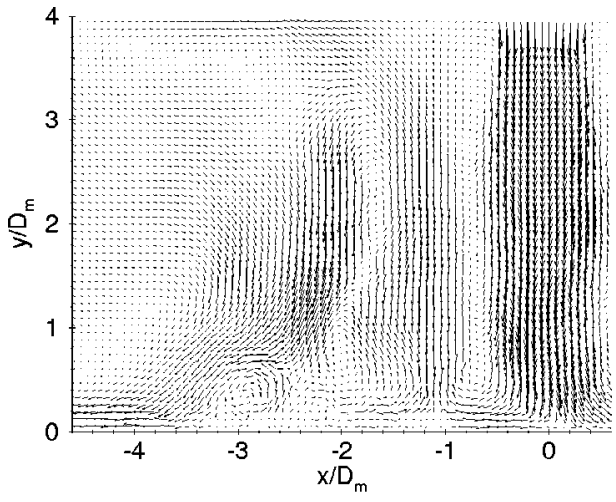

(a) snapshot 1

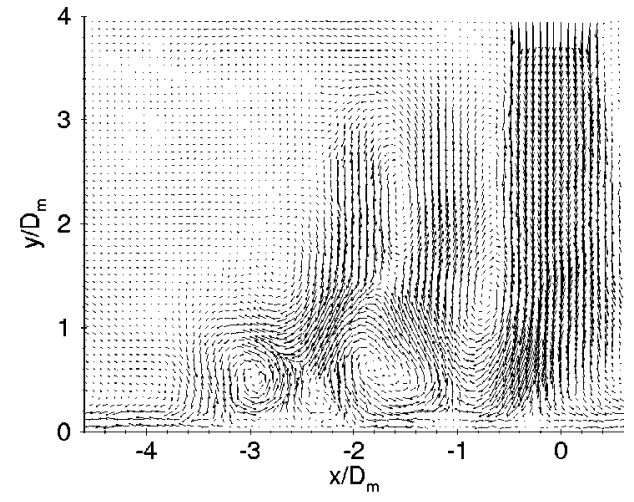

(b) snapshot 2
FIG. 8. POD filtered snapshots (mean field and the first 20 modes) in vertical plane 2 of the hexagonal arrangement. planes show that the flow is inherently unstable and that the energetic low mode number vortical structure oscillates from one to another neighbor jet.

The POD filtered snapshots in the horizontal plane at $0.25 D_{m}$ above the impingement plate are presented in Fig. 10. They reveal that the points where three wall jets collide are very unstable, in the sense that they do not have fixed positions. Figures 10(a)-10(c) show swirling motion appearing at some of these collision points. This motion constitutes a strong structure in terms of spatial energy, because it originates from one of the lower modes (mode 9) of the POD presented in Fig. 10(e). It is conjectured here that the eddies are formed by the unstable collision of three wall jets in one point. Naturally present fluctuations in the direction of the wall jets can cause the eddies in their collision points to rotate in either direction. This is illustrated by Fig. 10(f), which shows the two possible orientations of the eddies in the collision point of three wall jets. As the probability for each orientation is equal, the mean velocity field does not show the eddies.

The snapshot in Fig 10(a) seems more or less conventional in the sense that it shows the impingement locations in a roughly hexagonal pattern. However, slight displacements from the geometrical centers of the orifices occur due to oscillations in the individual jets. This is in line with the observations made from the POD filtered snapshots in the vertical planes.

\section{PODs of the in-line configuration}

The POD filtered snapshots in vertical planes $a$ and $b$ essentially showed stable impinging jets with a very short upwash flow at the position where their wall jets meet. Because the orifice pitch $s / D_{m}$ of the in-line configuration is twice as large as the pitch of the hexagonal configuration, the wall jets spread out over a greater surface area causing the upwash to be less strong, but more regular and located in or very close to the center between the two jets. In the in-line configuration no horse-shoe-like structures were found, most likely due to the higher pitch. Because the flow in these planes is very similar to the ensemble averaged flow field, no snapshots of planes $a$ and $b$ will be shown here.

More intriguing are the snapshots in vertical plane $c$ in the in-line configuration shown in Fig. 11. The shear layers of the jet at $x / D_{m}=0.0$ show strong fluctuations. In Fig. 11(a) there is a strong downflow on the right side of the jet, while in Fig. 11(b) it is on the left. This indicates a swinging motion of the jet. The jet at $x / D_{m}=4.0$ appears undisturbed in both snapshots of Fig. 11, because it is the least disturbed while it has fewer neighbors to interact with, compared to the other jets.

In Fig. 12 POD filtered snapshots are presented in the horizontal plane at $0.23 D_{m}$ above the impingement plate for the in-line configuration. At $\left(x / D_{m}, z / D_{m}\right) \approx(-0.1,3.1)$ there is a cluster of three vectors that are significantly shorter than

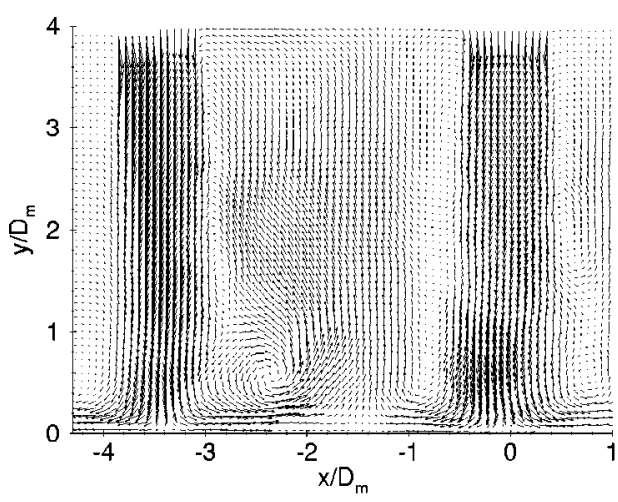

(a) snapshot 1

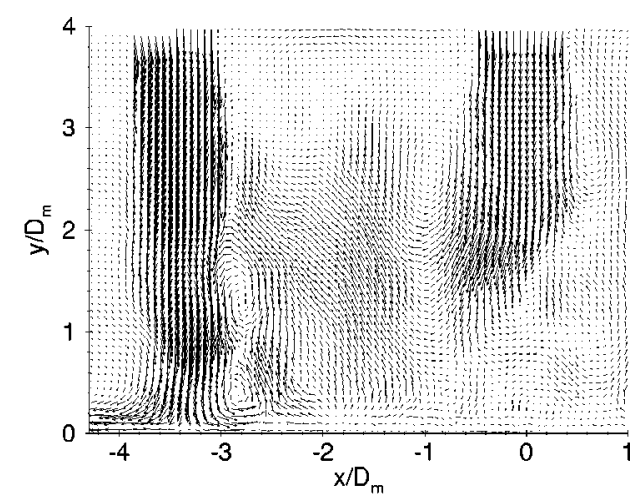

(b) snapshot 2
FIG. 9. POD filtered snapshots (mean field and the first 20 modes) in vertical plane 3 of the hexagonal arrangement. 


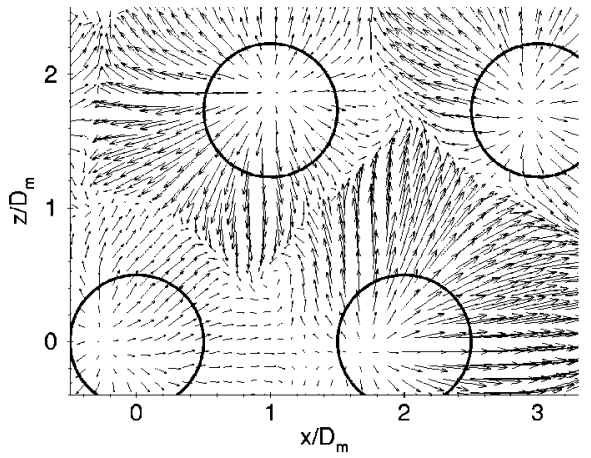

(a) snapshot 1

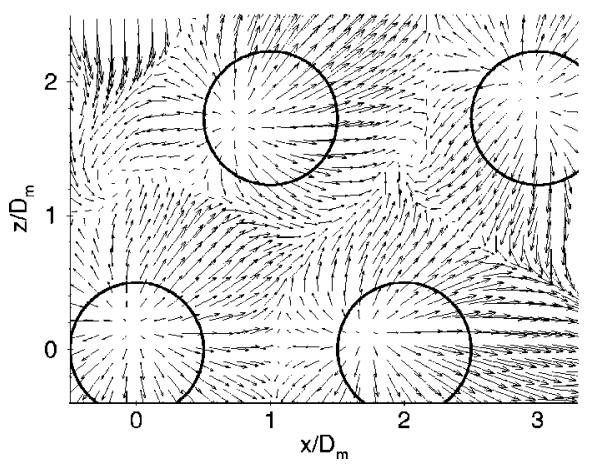

(c) snapshot 3

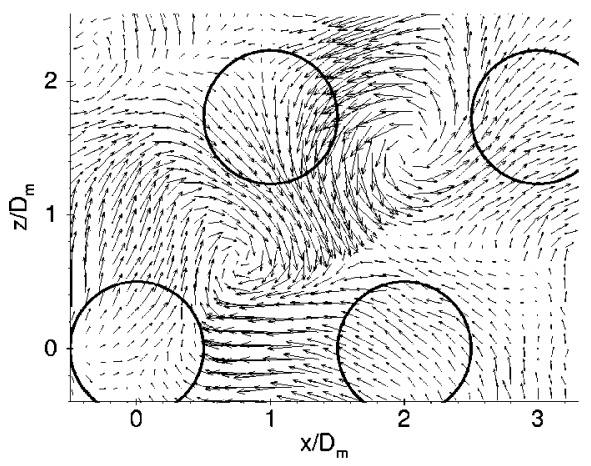

(e) POD mode 9

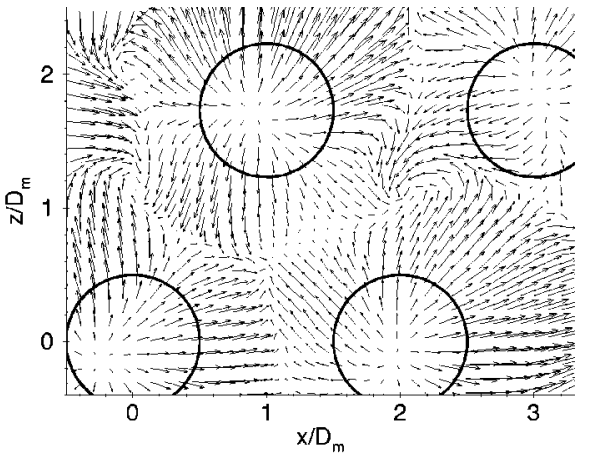

(b) snapshot 2

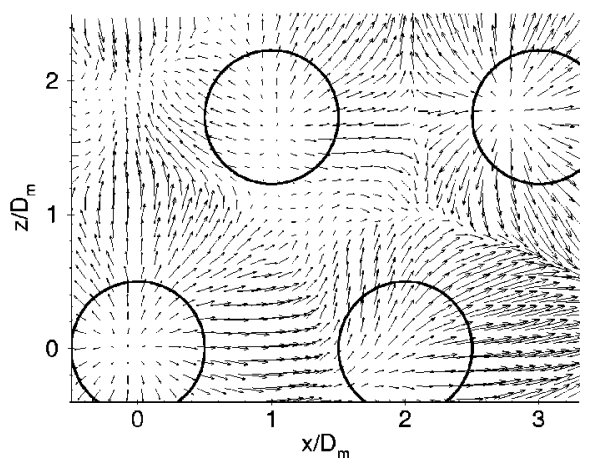

(d) snapshot 4

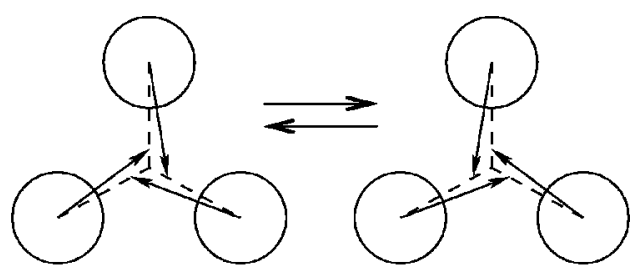

(f) Instable wall jet collision
FIG. 10. POD filtered snapshots (mean field and the first 20 modes) and the ninth mode in the horizontal plane at $0.25 D_{m}$ above the impingement plate of the hexagonal arrangement. the surrounding vectors. These are errors introduced by the fouling of the impingement plate by impinging seeding particles, as a result of the PIV measurement system configuration. The stagnation points of the jets show some oscillation, though not as strong as for the hexagonal configuration.

There is no point where four wall jets collide. Instead there appear to be two points where three wall jets meet, connected by a line where two wall jets meet. This is illustrated by Fig. 12(d). The drawing on the left shows the four orifices under investigation and the wall jets they produce. Intuitively, the wall jets are expected to meet in (or at least near) the geometrical center of the four jet impingement points. Instead, near the geometrical center a region exists where the wall jets of the center and the outer jet meet (the dashed line) and at either end of this region there is a collision point of three wall jets, schematically depicted in the right drawing of Fig. 12(d).

A second interesting feature is the occurrence of vortex- like structures at some of the collision points of three wall jets, similar to the snapshots in the horizontal plane of the hexagonal configuration. They are clearly visible in Figs. 12 (a) and $12(\mathrm{~b})$ around $\left(x / D_{m}, z / D_{m}\right) \approx(-1,2.1)$ and $(0.6,2.2)$

\section{E. Vortex size distribution}

The $Q$ criterion can be used as a vortex identifier to the POD filtered snapshots. This has the advantage that smallscale disturbances do not interfere with the location of large eddies. An example of $Q$ maps associated with the raw snapshot and their POD filtered equivalent (presented in Fig. 6) can be found in Fig. 13. Comparing Fig. 13(b) with Fig. 13(a), it is clear that the structures in the POD filtered snapshot are larger than the ones in the original snapshot. Next to that, a lot of smaller vortices in the original snapshot do not appear in the POD filtered one. The most energetic structures 


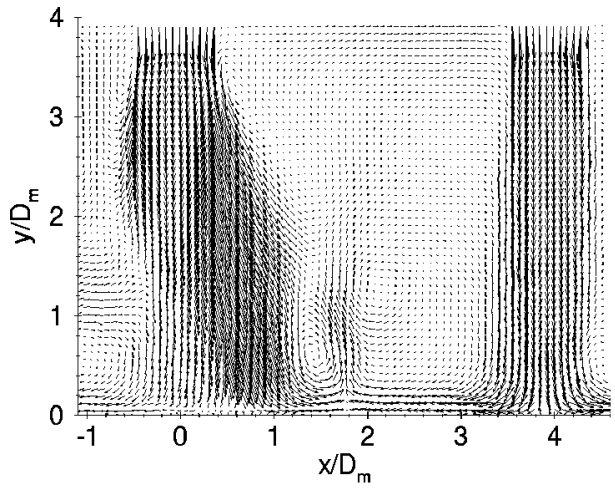

(a) snapshot 1

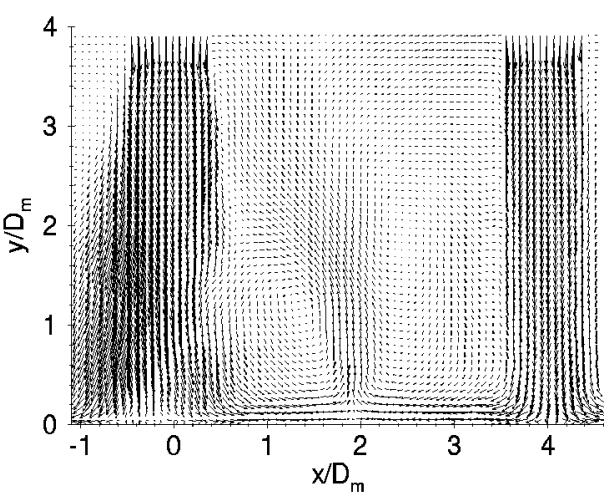

(b) snapshot 2
FIG. 11. POD filtered snapshots (mean field and the first 20 modes) in vertical plane $c$ of the in-line arrangement. are trapped by the identification method after POD filtering, whereas smaller and less energetic structures are ignored.

For a more quantitative investigation of the large eddies, $Q$ maps of POD filtered snapshots are used to locate and determine their size. By choosing an appropriate threshold level for $Q$, individual eddies can be isolated and their position can be determined using

$$
x_{e}=\frac{\iint_{A} x Q(x, y) d x d y}{\iint_{A} Q(x, y) d x d y}
$$

and

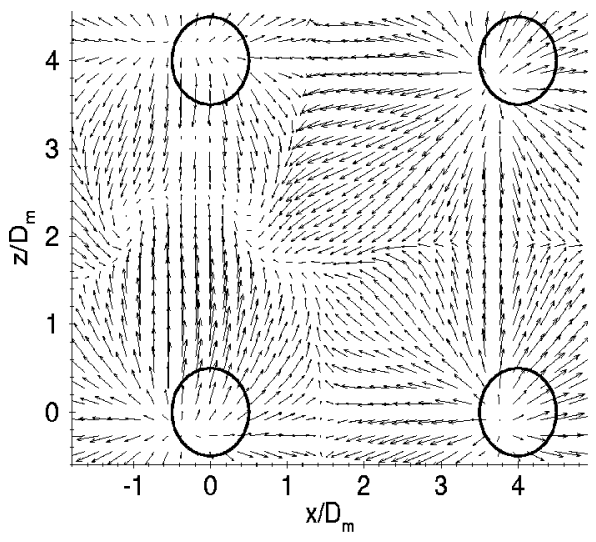

(a) snapshot 1

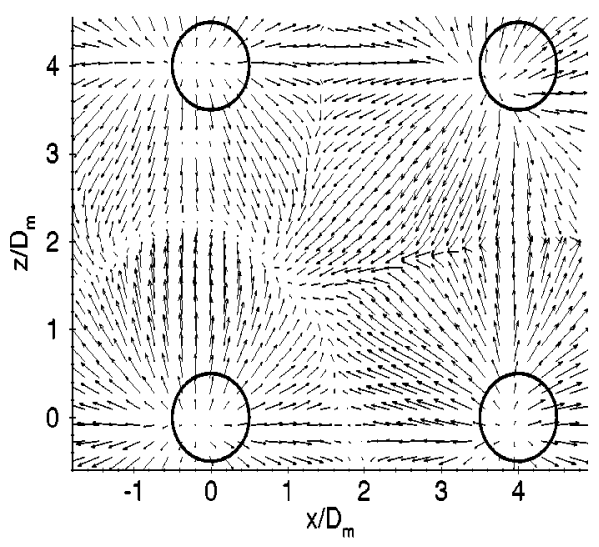

(c) snapshot 3

$$
y_{e}=\frac{\iint_{A} y Q(x, y) d x d y}{\iint_{A} Q(x, y) d x d y},
$$

where $x_{e}$ and $y_{e}$ denote the position of the center of an eddy, and $A$ is the area enclosing the eddy [i.e., where $Q(x, y)$ exceeds the threshold level].

Because the shape of an eddy identified with $Q$ can be quite irregular, there is no unique way to measure its diameter. For this reason a characteristic diameter $D_{e}$ is calculated on the basis of the total area enclosed by the eddy:

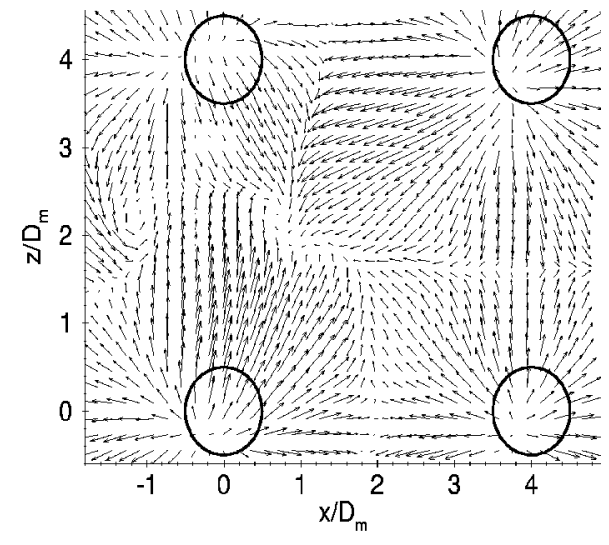

(b) snapshot 2

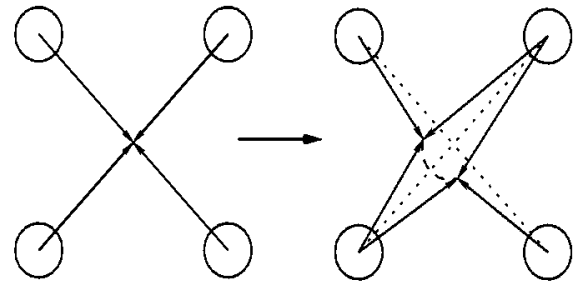

FIG. 12. POD filtered snapshots (mean field and the first 20 modes) in the horizontal plane at $0.23 D_{m}$ above the impingement plate of the in-line arrangement. (d) Schematic of colliding wall jets 


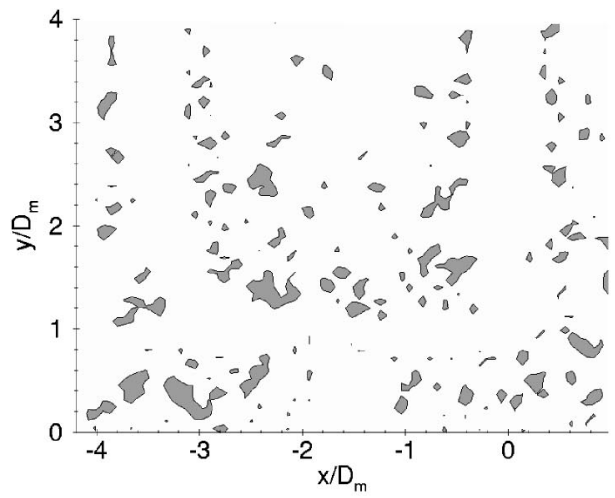

(a) Original snapshot

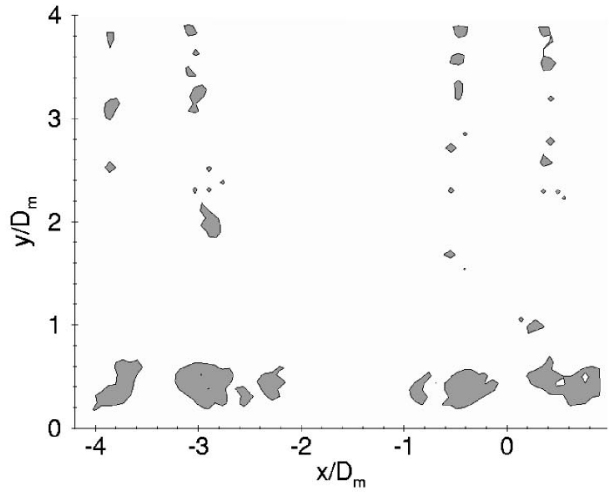

(b) POD filtered snapshot
FIG. 13. Comparison of the eddies found in a snapshot in plane 3 of the hexagonal configuration and its POD filtered equivalent with threshold $Q D_{m}^{2} / U_{C L}^{2}=1.0$.

$$
D_{e}=\sqrt{\iint_{A} d x d y}
$$

It should be noted that this diameter strongly depends on the chosen threshold value for $Q$. Therefore, it is only to be used for deriving qualitative conclusions regarding the size distribution of eddies.

Finally, the positions of the eddies, ordered according to their size, are presented in graphs. As an example, Fig. 14 presents the distributions of eddies in two size classes, ranging from $0.1 D_{m}$ to $0.3 D_{m}$, identified in an ensemble of 1000 POD filtered snapshots in vertical plane 3 of the hexagonal configuration with sharp-edged orifices. Each dot represents an eddy.

In Fig. 14(a) the eddies are concentrated in the shear layer and near the impingement points. In the downstream direction of the jets, the spreading of the shear layers is clear from the broadening of the regions of eddies. The highest eddy density is found in the direct neighborhood of the impingement points. This is the area where vortices originating from the shear layers impinge on the wall and are possibly destroyed.

It appears from Fig. 14(b) that the larger eddies are found just above the wall, next to the impingement points of the jets. The highest concentration of large eddies is found at the position of the horse-shoe vortex that circumscribes the outer jet. Eddy size distributions in other vertical planes in the hexagonal and the in-line configurations were very similar to the presented distributions and will not be presented here.

\section{CONCLUSIONS}

Results were presented for the vorticity magnitude $\left|\omega_{z}\right|$, the second invariant of the velocity gradient tensor $Q$, and the kinematic vorticity number $N_{k}$ in several planes of two configurations of multiple impinging jets. These three techniques were compared for their applicability to the identification of eddies. It appeared that $Q$ is the most adequate technique of the three, because $\left|\omega_{z}\right|$ does not distinguish vortices from background shear and $N_{k}$ appeared to be, at least for the flow considered, oblivious to the dynamical significance of a vortex.

POD is subsequently presented as a technique to apply inhomogeneous low-pass filtering of snapshots to reveal the large-scale dynamics of the flow snapshots resulting from PIV measurements. From reconstructed snapshots on the basis of the 21 most energetic modes the large-scale dynamics of the jets are subsequently investigated.

For the hexagonal configuration the jets appear to be largely unaffected down to $y=2 D_{m}$, but below this position the jets were disturbed by eddies originating from the interaction of the jets with the impingement surface. These eddies also cause the upwash flows between jets to oscillate, which in turn affects their interaction with the impinging jets. In the

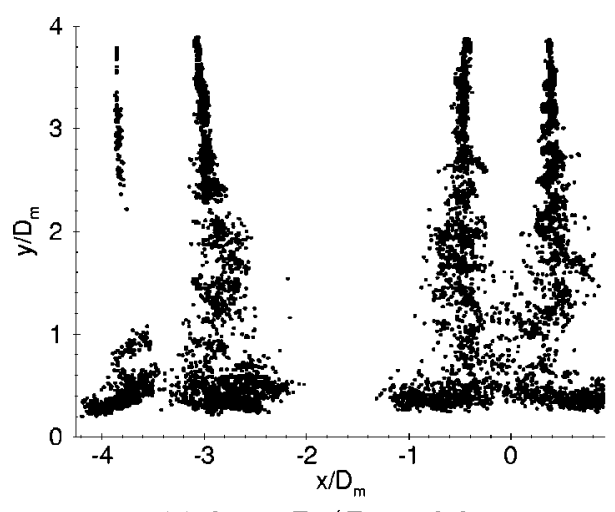

(a) $0.1<D_{e} / D_{m} \leq 0.2$

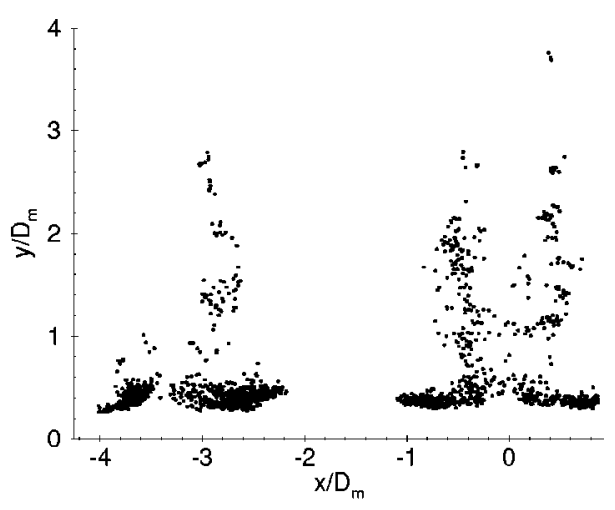

(b) $0.2<D_{e} / D_{m} \leq 0.3$
FIG. 14. Size distribution of eddies located in vertical plane 3 of the hexagonal configuration. 
POD filtered snapshots in vertical plane 3 clear evidence was presented of the horse-shoe vortex around the outer jet. This vortex did not have a steady position near the outer jet. The POD filtered snapshots in the horizontal plane were presented. It was conjectured that vortical motion between the impingement points of three adjacent jets is caused by the unstable collision of the three wall jets in that point. As both orientations of the eddies can appear equally likely, the mean velocity field did not show the eddies.

Due to the larger pitch in the in-line configuration, the upwash between the jets was shorter than the one in the hexagonal configuration. As a result, the interaction between the jets was less and they appeared much more stable in comparison to the hexagonal configuration. The lack of a horse-shoe vortex around the outer jets in the in-line configuration was most likely caused by the higher pitch. The distortion of the neighbors of the center jet was clearly visible. The jets appeared to be swinging, causing strong fluctuations in its shear layer. The outer jet appeared undisturbed. In the horizontal snapshots in the in-line configuration there appeared to be no points of collision for four wall jets. Contrary to intuition, there were two collision points of three wall jets connected by a collision line of two wall jets. In the two collision points vortices can exist, but there is a clear preference for one of the two points. The existence of a vortex in one of the collision points inhibits the appearance of a second vortex in the other one.

On the basis of $Q$ maps of the POD filtered snapshots in vertical plane 3 of the hexagonal configuration, eddies were identified. Subsequently, the eddies were arranged in four size classes that were presented in graphs. The larger eddies were found just above the impingement wall in the vicinity of the jet impingement points, and at the position of the horse-shoe vortex circumscribing the outer jet.

\section{ACKNOWLEDGMENTS}

This work was sponsored by the Technology Foundation STW of the Netherlands, TNO-TPD, and Rademaker-Den Boer.

${ }^{1} \mathrm{~K}$. Kataoka, "Impingement heat transfer augmentation due to large scale eddies," Heat Transfer 1990, Proceedings of the Ninth International Heat Transfer Conference, January 1990, edited by G. Hetsroni (Hemisphere, New York, 1990), Vol. 1, p. 255-273.

${ }^{2}$ S. Bae and H.-J. Sung, "Breakdown of the Reynolds analogy in a stagnation region under inflow disturbances," Theor. Comput. Fluid Dyn. 14, 377 (2001).

${ }^{3}$ J. Jeong and F. Hussain, "On the identification of a vortex," J. Fluid Mech. 285, 69 (1995).

${ }^{4}$ J. C. Hunt, A. A. Wray, and P. Moin, "Eddies, stream and convergence zones in turbulent flows," Proceedings of the 1988 Summer Program (NASA Ames/Stanford University, Center for Turbulence Research, Stanford, CA, 1988), Report No. CTR-S88, pp. 193-208.

${ }^{5}$ M. S. Chong, A. E. Perry, and B. J. Cantwell, "A general classification of three-dimensional flow fields," Phys. Fluids A 2, 765 (1990).

${ }^{6}$ R. J. Adrian, K. T. Christensen, and Z.-C. Liu, "Analysis and interpretation of instantaneous turbulent velocity fields," Exp. Fluids 29, 275 (2000).

${ }^{7}$ S. Gamard, W. K. George, D. Jung, and S. Woodward, "Application of a "slice" proper orthogonal decomposition to the far field of an axisymmetric turbulent jet," Phys. Fluids 14, 2515 (2002).

${ }^{8} \mathrm{M}$. Manhart and H. Wengle, "A spatiotemporal decomposition of a fully inhomogeneous turbulent flow field," Theor. Comput. Fluid Dyn. 5, 223 (1993).

${ }^{9}$ L. Sirovich, "Turbulence and the dynamics of coherent structures: I, II, III," Q. Appl. Math. 45, 561 (1987).

${ }^{10}$ R. D. Mehta and P. Bradshaw, "Design rules for small low speed wind tunnels," Aeronaut. J. 73, 443 (1979). Technical note.

${ }^{11}$ R. Matsumoto, I. Ishihara, T. Yabe, K. Ikeda, S. Kikkawa, and M. Senda, "Impingement heat transfer within arrays of circular jets including the effect of crossflow (AJTE99-6386)," Proceedings of the Fifth ASME/ JSME Joint Thermal Eng. Conference, March 1999.

${ }^{12}$ L. F. G. Geers, M. J. Tummers, and K. Hanjalić, "Experimental investigation of impinging jet arrays," Exp. Fluids 36, 946 (2004). 